\title{
SOLUTION OF THE $\gamma$-SPACE PROBLEM
}

\section{RALPH FOX}

ABSTRACT. This paper disproves the classic conjecture that every $\gamma$-space is quasimetrizable.

A quasi-metric on a set $X$ is a generalized metric $d: X \times X \rightarrow[0, \infty)$ satisfying the axioms $d(x, y)=0 \Leftrightarrow x=y$ and $d(x, z) \leqslant d(x, y)+d(y, z)$, but not necessarily the axiom of symmetry [N, W]. As with a metric, the family of all sets $B_{d}(x, r)=\{y: d(x, y)<r\}$, for $r>0$, form a neighbourhood base at each $x \in X$ for a topology on $X$. A space $X$ with such a topology is called quasi-metrizable.

Following [J1], a (an open) neighbournet $V$ on a space $X$ is a binary relation on $X$ such that, for each $x \in X$, the set $V[x]$ is a (an open) neighbourhood of $x$. A neighbournet $V$ is called a normal neighbournet if there exists a sequence $\left\langle V_{k}\right.$ : $k \in \mathbf{N}\rangle$ of neighbournets with $V_{k+1}^{2} \subseteq V_{k}$ for each $k \in \mathbf{N}$ and with $V_{1} \subseteq V$. Clearly, if $X$ is a quasi-metrizable space with quasi-metric $d$, and if for some $n \in \mathbf{N}$ and each $x \in X$ we have $B_{d}(x, 1 / n) \subseteq V[x]$, then $V$ is a normal neighbournet; for we may define the $V_{k}$ by $V_{k}[x]=B_{d}\left(x, 2^{-k} / n\right)$.

With the above terminology, a $T_{1}$ space $X$ is called a $\gamma$-space if there exists a decreasing sequence $\left\langle V_{k}: k \in \mathbf{N}\right\rangle$ of neighbournets (called a $\gamma$-sequence) such that, for each $x \in X$, the family $\left\{V_{k}^{2}[x]: k \in \mathbf{N}\right\}$ is a neighbourhood base at $x$ [LF, J1]. Clearly every quasi-metrizable space is a $\gamma$-space since we may define the $V_{k}$ by $V_{k}[x]=B_{d}\left(x, 2^{-k}\right)$.

The question as to whether every $\gamma$-space is quasi-metrizable has been raised frequently (and often independently) in the literature, for example [NČ, S, LN, LF, $\mathbf{G}, \mathbf{J 2}$ ], and is listed as Classic Problem VIII in [TP]. Indeed, a proof of this question was first claimed in the literature in 1943 [R1, p. 35]; however the argument given was incomplete and the author had later to strengthen his required conditions [R2]. Currently several partial solutions have been obtained [G-K2; J2; B-K1; F2].

In this paper we construct a Hausdorff counterexample to the $\gamma$-space conjecture (currently we know of no regular counterexample). If $U^{n}$ is a normal neighbournet whenever $U$ is a neighbournet on $X$, then the space $X$ is said to be $n$-pretransitive [FL]. Our starting point will be the existence for each $n \in \mathbf{N}$, as proven in [F1], of a Hausdorff quasi-metrizable space $X_{n}$ which is not $n$-pretransitive (such a space is the $(n+1)$ th power $M^{n+1}$ of the Michael line $\left.M\right)$. Let $U_{n}$ be an open neighbournet on

Received by the editors July 23, 1981 and, in revised form, September 15, 1981.

1980 Mathematics Subject Classification. Primary 54E15; Secondary 54G20, 54E99.

Key words and phrases. Quasi-metric, $\gamma$-space, normal neighbournet, $n$-pretransitive space.

(c1982 American Mathematical Society 0002-9939/81/0000-1082/\$01.75 
$X_{n}$ such that $U_{n}^{n}$ is not a normal neighbournet. If we let $X=\cup_{n=1}^{\infty} X_{n}$ be the disjoint topological sum, and define the open neighbournet $U$ on $X$ by $U=\cup_{n=1}^{\infty} U_{n}$, then for no $k \in \mathrm{N}$ is $U^{k}$ a normal neighbournet. On the other hand each $X_{n}$, and hence $X$, are quasi-metrizable spaces and thus $\gamma$-spaces. From the space $X$ and open neighbournet $U$ we construct the non-quasi-metrizable $\gamma$-space $\tilde{X}$ as follows.

The points of $\tilde{X}$ are the points of $X \cup X^{2} \cup X^{3} \cup \cdots \cup X^{\omega}$. The basic open sets of $\tilde{X}$ are all sets in $X^{n}$ of the form $\left\{\left\langle x_{1}, \ldots, x_{n-1}\right\rangle\right\} \times A$ where $A$ is open in $X$, for each $n \in \mathbf{N}$, together with all sets

$$
\tilde{U}_{x ; k}=\{x\} \cup \bigcup_{n=k}^{\infty}\left(\left\{\left\langle x_{1}, \ldots, x_{n-1}\right\rangle\right\} \times U^{n-k+1}\left[x_{n}\right]\right)
$$

where $k \in \mathbf{N}$ and $x=\left\langle x_{1}, x_{2}, \ldots\right\rangle \in X^{\omega}$.

To show that $\tilde{X}$ is a $\gamma$-space, let $\left\langle V_{k}: k \in \mathbf{N}\right\rangle$ be a $\gamma$-sequence for $X$ with $V_{1} \subseteq U$ (for example, let $\left\langle W_{k}: k \in \mathbf{N}\right\rangle$ be any $\gamma$-sequence for $X$ and take $V_{k}=W_{k} \cap U$ ). Define neighbournets $\tilde{V}_{k}$ on $\tilde{X}$ as follows: If $x=\left\langle x_{1}, \ldots, x_{n}\right\rangle \in X^{n}$ then $\tilde{V}_{k}[x]=$ $\left\{\left\langle x_{1}, \ldots, x_{n-1}\right\rangle\right\} \times V_{k}\left[x_{n}\right]$, while if $x \in X^{\omega}$ then $\tilde{V}_{k}[x]=\tilde{U}_{x ; k}$. Then $\left\langle\tilde{V}_{k}: k \in \mathbf{N}\right\rangle$ is a $\gamma$-sequence for $\tilde{X}$. For if $x=\left\langle x_{1}, \ldots, x_{n}\right\rangle \in X^{n}$ then clearly the sets $\left(\tilde{V}_{k}\right)^{2}[x]=$ $\left\{\left\langle x_{1}, \ldots, x_{n-1}\right\rangle\right\} \times V_{k}^{2}\left[x_{n}\right]$, for $k \in \mathbf{N}$, form a neighbourhood base at $x$. Alternatively, if $x=\left\langle x_{1}, x_{2}, \ldots\right\rangle \in X^{\omega}$ then the sets

$$
\begin{aligned}
\left(\tilde{V}_{k}\right)^{2}[x] & =\{x\} \cup \bigcup_{n=k}^{\infty}\left(\left\{\left\langle x_{1}, \ldots, x_{n-1}\right\rangle\right\} \times V_{k} \circ U^{n-k+1}\left[x_{n}\right]\right) \\
& \subseteq\{x\} \cup \bigcup_{n=k}^{\infty}\left(\left\{\left\langle x_{1}, \ldots, x_{n-1}\right\rangle\right\} \times U^{n-k+2}\left[x_{n}\right]\right) \subseteq \tilde{V}_{k-1}[x],
\end{aligned}
$$

for $k \in \mathbf{N}$, form a neighbourhood base at $x$.

To show that $\tilde{X}$ is not quasi-metrizable, suppose $d$ is a quasi-metric for $\tilde{X}$ and define $x_{n}, z_{n} \in X$ by induction on $n \in \mathbf{N}$ as follows.

Assume inductively that $x_{1}, \ldots, x_{n-1}$ have been defined. Since $\left\{\left\langle x_{1}, \ldots, x_{n-1}\right\rangle\right\} \times$ $X \subseteq X^{n} \subseteq \tilde{X}$ is canonically homeomorphic to $X$, we may choose $x_{n}, z_{n} \in X$ such that $d\left(\left\langle x_{1}, \ldots, x_{n-1}, x_{n}\right\rangle,\left\langle x_{1}, \ldots, x_{n-1}, z_{n}\right\rangle\right)<1 / n$ but $z_{n} \notin U^{n}\left[x_{n}\right]$; for otherwise $U^{n}$ would be a normal neighbournet.

Having completed the induction, let $x=\left\langle x_{1}, x_{2}, \ldots\right\rangle \in X^{\omega}$. Find $m \in \mathbf{N}$ such that $B_{d}(x, 2 / m) \subseteq \tilde{U}_{x ; 1}$. Choose $n \geqslant m$ such that $d\left(x,\left\langle x_{1}, \ldots, x_{n}\right\rangle\right)<1 / m$; then $d\left(\left\langle x_{1}, \ldots, x_{n}\right\rangle,\left\langle x_{1}, \ldots, x_{n-1}, z_{n}\right\rangle\right)<1 / n \leqslant 1 / m$ but $d\left(x,\left\langle x_{1}, \ldots, x_{n-1}, z_{n}\right\rangle\right) \geqslant 2 / m$ since $\left\langle x_{1}, \ldots, x_{n-1}, z_{n}\right\rangle \notin \tilde{U}_{x ; 1}$. Thus $d$ does not satisfy the triangle inequality. This proves that the space $\tilde{X}$ is not quasi-metrizable.

\section{REFERENCES}

[B] H. R. Bennett, Quasi-metrizability and the $\gamma$-space property in certain generalized ordered spaces, Topology Proc. 4 (1979), 1-12.

[FL] P. Fletcher and W. F. Lindgren, Quasi-uniform spaces, Dekker, New York, 1982.

[F1] R. Fox, Pretransitivity and products of suborderable spaces, Topology and Order Structures, Part I, MC Tract 142, Math Centrum, Amsterdam, 1981.

[F2] , On metrizability and quasi-metrizability (to appear).

[G] G. Gruenhage, A note on quasi-metrizability, Canad. J. Math. 29 (1977), 360-366.

[J1] H. Junnila, Neighbornets, Pacific J. Math. 76 (1978), 83-108. 
[J2] _ Covering properties and quasi-uniformities of topological spaces, Ph.D. thesis, Virginia Polytech. Inst. and State Univ., Blacksburg, 1978.

[K1] J. Kofner, Transitivity and the $\gamma$-space conjecture in ordered spaces, Proc. Amer. Math. Soc. 81 (1981), 629-635.

[K2] _ Transitivity and orthobases, Canad. J. Math. (to appear).

[LF] W. F. Lindgren and P. Fletcher, Locally quasi-uniform spaces with countable bases, Duke Math. J. 41 (1974), 231-240.

[LN] W. F. Lindgren and P. Nyikos, Spaces with bases satisfying certain order and intersection properties, Pacific J. Math. 66 (1976), 455-476.

[NČ]. S. Nedev and M. Čoban, On the theory of o-metrizable spaces. III, Vestnik Moskov. Univ. Ser. I Mat. Meh. 27 (1972), no. 3, 10-15.

[N] V. V. Niemytzki, On the third axiom of metric space, Trans. Amer. Math. Soc. 29 (1927), 507-513.

[R1] H. Ribeiro, Sur les espaces à métrique faible, Portugal. Math. 4 (1943), 21-40.

[R2] __ Corrections à la note "Sur les espaces à métrique faible", Portugal. Math. 4 (1943), 65-68.

[S] R. Sabella, Convergence properties of neighboring sequences, Proc. Amer. Math. Soc. 38 (1973), 405-409.

[TP] Topology Proc. 2 (1977), 687-688.

[W] W. A. Wilson, On quasi-metric spaces, Amer. J. Math. 53 (1931), 675-684.

Department of Mathematics, SOUTHERn Illinois University at Carbondale, Carbondale, ILLINOIS 62901 